\title{
Preliminary Discussion on Several Problems Related to the Divisor Function
}

\section{Yongmin Wang*}

Binzhou Huimin Bureau of Yellow River Affairs, Binzhou, Shandong Province 251700, China. E-mail: 15866667538@163.com

Abstract: This paper is divided into three parts to discuss the divisor function. It mainly combines the high power sum of the divisor function to study the solution number of the polynomial with integer coefficients " $g(x) \equiv 0($ modq) “, and to improve some of the conclusions in the second chapter of Additive Theory of Prime Numbers and prove the improved conclusions.

Keywords: Divisor Function; Prove; Conclusion

\section{Introduction}

This article will mainly prove the following conclusions.

1.1 Research on the high power sum of the divisor function

Theorem 1: If $q$ is large enough, $\varepsilon$ is an arbitrarily small positive number, and $l$ is a positive integer,

1) When $l=3$, $\left.\exp \left(-0.84 \times\left(\frac{l}{\log 2}\right)^{l}\right) \cdot \log ^{2^{l}} q<\sum_{1 \leq n \leq q} \frac{d^{l}(n)}{n}<\exp \left(-2^{l-1} \cdot \log 2 \pi\right)+\varepsilon\right) \cdot \log ^{2^{2}} q$

2) When $l \geq 4$,

$\left.\exp \left(-0.59 \times\left(\frac{l}{\log 2}\right)^{l}\right) \cdot \log ^{2^{l}} q<\sum_{1 \leq n \leq q} \frac{d^{l}(n)}{n}<\exp \left(-2^{l-1} \cdot \log 2 \pi\right)+\varepsilon\right) \cdot \log ^{2^{l}} q$

\subsection{Research on the solution number of the polynomial with integer coefficients} " $g(x) \equiv 0($ modq $)$ "

Theorem 2: If $g(x)=b_{k} x^{k}+b_{k-1} x^{k-1}+\cdots+b_{1} x+b_{0}$ satisfies the condition $\left(b_{k}, \cdots b_{1}, b_{0}, q\right)=1$, then the solution number of $g(x) \equiv 0(\bmod q) \leq 2^{\pi(2 \mathrm{k})} \cdot q^{1-\frac{1}{k}}$

\subsection{Improvements to the conclusions of Chapter 2 in Additive Theory of Prime Numbers}

Theorem 3: Let $f\left(x_{1}, x_{2}, \cdots, x_{n}\right)$ represent a polynomial of degree $k$, whose coefficients are integers. Assuming that the greatest common divisor of all coefficients is 1 , then when $1 \leq x_{1}, x_{2}, \cdots, x_{n} \leq q$, the number of solutions of “ $f\left(x_{1}, x_{2}, \cdots, x_{n}\right) \equiv 0(\bmod q) " \leq 2^{n \pi(2 \mathrm{k})} \cdot q^{1-\frac{1}{k}} \cdot d^{n-1}(q)$.

Theorem 4: Let $f\left(x_{1}, x_{2}, \cdots, x_{n}\right)$ represent a polynomial of degree $k$, whose coefficients are integers. Assuming that the greatest common divisor of all coefficients is 1 , then

$$
\sum_{x_{1}-1}^{p} \cdots \sum_{x_{n}-1}^{p} d^{l}\left(\left|f\left(x_{1}, x_{2}, \cdots, x_{n}\right)\right|\right) \leq \mathrm{c}^{\frac{n}{k}} \cdot c_{1}(k, n, l) p^{n}(\log c p)^{c_{2}(k, n, l)}
$$

This is an open-access article distributed under the terms of the Creative Commons Attribution Non-Commercial License (http://creativecommons. org/licenses/by-nc/4.0/), which permits unrestricted non-commercial use, distribution, and reproduction in any medium, provided the original work is properly cited. 
The value of " $c$ " is the maximum value of the absolute value of each coefficient in $f\left(x_{1}, x_{2}, \cdots, x_{n}\right)$.

Note: Unless otherwise specified in all conclusions and proofs in the text, " $\log n$ " means " $\ln n$ ".

\section{Research on the high power sum of the divisor function}

An upper bound estimation result of “ $\sum_{1 \leq n \leq q} d^{l(\mathrm{n})}$ ” or “ $\sum_{1 \leq n \leq q} d^{l(\mathrm{n})} / n$ ” has been cited many times in Professor Hua Luogeng's Additive Theory of Prime Numbers, Number Theory Guide and Professor Pan Chengdong's Analytical Number Theory Basics, Goldbach Conjecture. It hasn't been found any expressions or references to their lower bound estimates. In order to better cite this valuation result and evaluate the influence of the errors generated in the citation process on the conclusion, this paper will give a lower bound valuation result.

Attachment: The conclusions given in Additive Theory of Prime Numbers are as follows ${ }^{[1]}$.

Lemma 2.4: Let $l$ be a positive integer, and then

$$
\sum_{1 \leq n \leq q} \frac{(d(n))^{l}}{n} \leq c_{6}(l) \cdot(\log \mathrm{q})^{2^{l}}
$$

Lemma 2.5 Let $l$ be a positive integer, and then

$$
\sum_{1 \leq n \leq q}(d(n))^{l} \leq c_{7}(l) \cdot(\log \mathrm{q})^{2^{l}-1}
$$

\subsection{In the following certification process, the following symbols will be cited}

1 “ $u(n)$ " means "Mobius" and " $\mid$ " means absolute value.

2 " ( ) $q$ " means the sum of the items whose denominator is not greater than " $q$ " after expanding the items in " ( )".

3 “ $\pi(n)$ " represents the number of prime numbers not greater than " $n$ ".

4" $P$ " represents prime numbers, and $P_{1}, P_{2}, \cdots, P_{n}$ represents all prime numbers not greater than $q$. $P_{i}<P_{i+1}(1 \leq i \leq s-1)$.

5Common symbols such as "

\subsection{The conclusions to be proved in this part}

Due to the special relationship between " $\sum_{1 \leq n \leq q} d^{l}(n)$ ” and " $\sum_{1 \leq n \leq q} d^{l}(n) / n$ ”, it is appropriate to study only the valuation of “ $\sum_{1 \leq n \leq q} d^{l}(n) / n$ ” in this article. According to relevant knowledge:

when $l=0, \sum_{1 \leq n \leq q} \frac{1}{n}=\log q+O(1)$;

when $l=1, \sum_{1 \leq n \leq q} d(n) / n=\frac{1}{2} \log ^{2} q+O(\log q)$;

when $l=2, \sum_{1 \leq n \leq q} d^{2}(n) / n=\frac{1}{4 \pi^{2}} \log ^{4} q+O\left(\log ^{3} \mathrm{q}\right)$.

Here this paper studies the situation when $l \geq 3$.

Theorem 1: If $q$ is large enough and $\varepsilon$ is any positive number greater than 0 , then:

when $l=3$,

$\exp \left(-0.84 \times\left(\frac{l}{\log 2}\right)^{l}\right) \cdot \log ^{2^{l}} q<\sum_{1 \leq n \leq q} \frac{d^{l}(n)}{n}<\left(\exp \left(-2^{l-1} \cdot \log 2 \pi\right)+\varepsilon\right) \cdot \log ^{2^{2}} q ;$

when $l \geq 4$,

$\exp \left(-0.59 \times\left(\frac{l}{\log 2}\right)^{l}\right) \cdot \log ^{2^{l}} q<\sum_{1 \leq n \leq q} \frac{d^{l}(n)}{n}<\left(\exp \left(-2^{l-1} \cdot \log 2 \pi\right)+\varepsilon\right) \cdot \log ^{2^{l}} q$.

\subsection{Proof of several lemmas}


Lemma 1: The aim is to prove that:
1) when $l=2, \sum_{1 \leq n \leq q} d^{l}\left(\mathrm{P}_{i}^{m}\right) / \mathrm{P}_{i}^{m} \leq \frac{4.87 \times\left(\frac{l}{\log 2}\right)^{l}}{\mathrm{P}_{i}^{2}}$;
2) when $l \geq 3, \sum_{1 \leq n \leq q} d^{l}\left(\mathrm{P}_{i}^{m}\right) / \mathrm{P}_{i}^{m} \leq \frac{5.71 \times\left(\frac{l}{\log 2}\right)^{l}}{\mathrm{P}_{i}^{2}}$.

The proving process is: set the function $f(x)=\frac{x^{l}}{P_{i}^{x}}\left(x, P, l\right.$ are not less than 2), then $f^{\prime}(x)=\left(l-x \log P_{i}\right) \bullet x^{l-1} / P_{i}^{x}$ . It is easy to know that: when $x>\frac{l}{\log P_{i}}, f(x)$ is a monotone decreasing function; when $x<\frac{l}{\log P_{i}}, f(x)$ is a monotone increasing function.

$$
\begin{aligned}
& \text { 1) When } l=2, \frac{l}{\log P_{i}} \leq \frac{2}{\log 2}<3 \\
& \sum_{m=2}^{\infty} d^{l}\left(\mathrm{P}_{i}^{m}\right) / \mathrm{P}_{i}^{m}=\sum_{m=2}^{\infty} \frac{(m+1)^{2}}{\mathrm{P}_{i}^{m}}=\frac{3^{2}}{\mathrm{P}_{i}^{2}}+P_{i} \sum_{m=4}^{\infty} \frac{m^{2}}{\mathrm{P}_{i}^{m}} \leq \frac{3^{2}}{P_{i}^{2}}+P_{i} \int_{3}^{\infty} \frac{x^{2}}{P_{i}^{x}} d x \\
& =\frac{3^{2}}{P_{i}^{2}}+\frac{1}{P_{i}^{2}}\left(\frac{3^{2}}{\log P_{i}}+\frac{3 \cdot 2}{\log ^{2} P_{i}}+\frac{2}{\log ^{3} P_{i}}\right) \leq \frac{3^{2}}{P_{i}^{2}}+\frac{1}{P_{i}^{2}}\left(\frac{3^{2}}{\log 2_{i}}+\frac{6}{\log ^{2} 2}+\frac{2}{\log ^{3} 2}\right) \\
& \leq \frac{4.87 \times\left(\frac{l}{\log 2}\right)^{l}}{P_{i}^{2}}(\text { It makes sense when } l=2)
\end{aligned}
$$

2) When $l \geq 3$, there are two cases for discussion.

$$
\begin{aligned}
& \text { (1) If } \frac{l}{\log P_{i}}>3, \sum_{m=2}^{\infty} d^{l}\left(\mathrm{P}_{i}^{m}\right) / \mathrm{P}_{i}^{m}=\mathrm{P}_{i} \sum_{m=2}^{\infty} \frac{(m+1)^{l}}{\mathrm{P}_{i}^{m+1}} \\
& \leq \mathrm{P}_{i}\left(\int_{3}^{\infty} \frac{x^{l}}{P_{i}^{x}} d x+\left(\frac{l}{\log P_{i}}\right)^{l} / \mathrm{P}_{i}^{\frac{l}{\log P_{i}}}\right) \\
& \leq\left(\frac{l}{\log P_{i}}\right)^{l} / \mathrm{P}_{i}^{2}+\mathrm{P}_{i} \int_{3}^{\infty} \frac{x^{l}}{P_{i}^{x}} d x\left(\text { use } \frac{l}{\log P_{i}} \geq 3\right) \\
& =\left(\frac{l}{\log P_{i}}\right)^{l} / \mathrm{P}_{i}^{2}+\frac{1}{\mathrm{P}_{i}^{2}}\left(\frac{3^{l}}{\log P_{i}}+\frac{3^{l-1} \cdot l}{\log ^{2} P_{i}}+\frac{3^{l-2} \cdot l \cdot(l-1)}{\log ^{3} P_{i}}+\cdots+\frac{l !}{\log ^{l+1} P_{i}}\right) \\
& \leq\left(\frac{l}{\log P_{i}}\right)^{l} / \mathrm{P}_{i}^{2}+\frac{l^{l}}{P_{i}^{2}}\left(\frac{1}{\log P_{i}}+\frac{1}{\log ^{2} P_{i}}+\frac{1}{\log ^{3} P_{i}}+\cdots+\frac{1}{\log ^{l+1} P_{i}}\right)(\text { use } l \geq 3) \\
& \leq \\
& \left(\frac{l}{\log P_{i}}\right)^{l} / \mathrm{P}_{i}^{2}+\frac{l^{l}}{P_{i}^{2}}\left(\frac{1}{\log 2}+\frac{1}{\log ^{2} 2}+\frac{1}{\log ^{3} 2}+\cdots+\frac{1}{\log ^{l+1} 2}\right)\left(\text { (use } P_{i} \geq 2\right) \\
& 5.71 \times\left(\frac{l}{\log 2}\right)^{l} \\
& \leq \frac{P_{i}^{2}}{}
\end{aligned}
$$

(2) When $\frac{l}{\log P_{i}}<3$, 


$$
\begin{aligned}
& \sum_{m=2}^{\infty} d^{l}\left(\mathrm{P}_{i}^{m}\right) / \mathrm{P}_{i}^{m}=d^{l}\left(\mathrm{P}_{i}^{2}\right) / \mathrm{P}_{i}^{2}+P_{i} \sum_{m=3}^{\infty} d^{l}\left(\mathrm{P}_{i}^{m}\right) / \mathrm{P}_{i}^{m+1} \\
& =d^{l}\left(\mathrm{P}_{i}^{2}\right) / \mathrm{P}_{i}^{2}+P_{i} \sum_{m \geq 4} \frac{m^{l}}{\mathrm{P}_{i}^{m}} \leq d^{l}\left(\mathrm{P}_{i}^{2}\right) / \mathrm{P}_{i}^{2}+P_{i} \int_{3}^{\infty} \frac{x^{l}}{P_{i}^{x}} d x \\
& \left.\leq \frac{3^{l}}{P_{i}^{2}}+\frac{l^{l}}{P_{i}^{2}}\left(\frac{1}{\log P_{i}}+\frac{1}{\log ^{2} P_{i}}+\frac{1}{\log ^{3} P_{i}}+\cdots+\frac{1}{\log { }^{l+1} P_{i}}\right) \text { (use } l \geq 3\right) \\
& \left.\leq \frac{3^{l}}{P_{i}^{2}}+\frac{4.71 \times\left(\frac{l}{\log 2}\right)^{l}}{P_{i}^{2}} \leq 5.71 \times\left(\frac{l}{\log 2}\right)^{l} / P_{i}^{2} \text { (use } \frac{l}{\log 2} \geq \frac{3}{\log 2}>3\right)
\end{aligned}
$$

Based on the conclusions obtained above, it can be seen that Lemma 1 makes sense. The proving process is completed.

Lemma 2: The aim is to prove that:

1) When $l=2, \sum_{1 \leq n \leq q} \frac{d^{l}(n)}{n} \leq \exp \left(2.44 \times\left(\frac{l}{\log 2}\right)^{l}\right) \cdot \sum_{1 \leq n \leq q}|\mathrm{u}(\mathrm{n})| \frac{d^{l}(n)}{n}$

2) When $l \geq 3, \sum_{1 \leq n \leq q} \frac{d^{l}(n)}{n} \leq \exp \left(2.86 \times\left(\frac{l}{\log 2}\right)^{l}\right) \cdot \sum_{1 \leq n \leq q}|\mathrm{u}(\mathrm{n})| \frac{d^{l}(n)}{n}$

The proving process is: from the definition of " ( ) $)_{q}$, it can be seen

$$
\begin{aligned}
& \sum_{1 \leq n \leq q} \frac{d^{l}(n)}{n}=\left(\prod_{i=1}^{s}\left(1+\frac{d^{l}\left(P_{i}\right)}{P_{i}}+\frac{d^{l}\left(P_{i}^{2}\right)}{P_{i}^{2}}+\frac{d^{l}\left(P_{i}^{3}\right)}{P_{i}^{3}}+\cdots+\frac{d^{l}\left(P_{i}^{m}\right)}{P_{i}^{m}}+\cdots\right)\right)_{q} \\
& =\left(\left(1+\frac{d^{l}\left(P_{1}\right)}{P_{1}}\right) \cdot \prod_{i=2}^{s}\left(1+\frac{d^{l}\left(P_{i}\right)}{P_{i}}+\frac{d^{l}\left(P_{i}^{2}\right)}{P_{i}^{2}}+\frac{d^{l}\left(P_{i}^{3}\right)}{P_{i}^{3}}+\cdots+\frac{d^{l}\left(P_{i}^{m}\right)}{P_{i}^{m}}+\cdots\right)\right)_{q} \\
& +\sum_{\alpha=2}^{\infty} \frac{d^{l}\left(P_{1}^{\alpha}\right)}{P_{1}^{\alpha}} \cdot\left(\prod_{i=2}^{s}\left(1+\frac{d^{l}\left(P_{i}\right)}{P_{i}}+\frac{d^{l}\left(P_{i}^{2}\right)}{P_{i}^{2}}+\frac{d^{l}\left(P_{i}^{3}\right)}{P_{i}^{3}}+\cdots+\frac{d^{l}\left(P_{i}^{m}\right)}{P_{i}^{m}}+\cdots\right)\right)_{q} / P_{1}^{\alpha} \\
& \leq\left(\left(1+\frac{d^{l}\left(P_{1}\right)}{P_{1}}\right) \cdot \prod_{i=2}^{s}\left(1+\frac{d^{l}\left(P_{i}\right)}{P_{i}}+\frac{d^{l}\left(P_{i}^{2}\right)}{P_{i}^{2}}+\frac{d^{l}\left(P_{i}^{3}\right)}{P_{i}^{3}}+\cdots+\frac{d^{l}\left(P_{i}^{m}\right)}{P_{i}^{m}}+\cdots\right)\right)_{q} \\
& +\sum_{\alpha=2}^{\infty} \frac{d^{l}\left(P_{1}^{\alpha}\right)}{P_{1}^{\alpha}} \cdot\left(\prod_{i=2}^{s}\left(1+\frac{d^{l}\left(P_{i}\right)}{P_{i}}+\frac{d^{l}\left(P_{i}^{2}\right)}{P_{i}^{2}}+\frac{d^{l}\left(P_{i}^{3}\right)}{P_{i}^{3}}+\cdots+\frac{d^{l}\left(P_{i}^{m}\right)}{P_{i}^{m}}+\cdots\right)\right)_{q} \\
& \leq\left(1+\sum_{\alpha=2}^{\infty} \frac{d^{l}\left(P_{\alpha}\right)}{P_{\alpha}}\right) \cdot\left(\left(1+\frac{d^{l}\left(P_{1}\right)}{P_{1}}\right) \cdot \prod_{i=2}^{s}\left(1+\frac{d^{l}\left(P_{i}\right)}{P_{i}}+\frac{d^{l}\left(P_{i}^{2}\right)}{P_{i}^{2}}+\cdots\right)\right)_{q} \\
& \leq \cdots \text { (repeat the derivation process of } P_{1} \text { for } " P_{i} "(2 \leq i \leq s) \text { ) } \\
& \leq \prod_{i=1}^{s}\left(1+\sum_{\alpha=2}^{\infty} \frac{d^{l}\left(P_{i}^{\alpha}\right)}{P_{i}^{\alpha}}\right) \cdot\left(\prod_{i=1}^{s}\left(1+\frac{d^{l}\left(P_{i}\right)}{P_{i}}\right)\right)_{q} \\
& \leq \prod_{i=1}^{s}\left(1+\frac{c}{P_{i}^{2}}\right) \cdot\left(\prod_{i=1}^{s}\left(1+\frac{d^{l}\left(P_{i}\right)}{P_{i}}\right)\right)_{q} \quad(\mathrm{c} \text { is a constant to be determined }) \\
& =\exp \left(\sum_{i=1}^{s} \log \left(1+\frac{d^{l}(\mathrm{c})}{P_{i}^{2}}\right)\right) \cdot\left(\prod_{i=1}^{s}\left(1+\frac{d^{l}\left(P_{i}\right)}{P_{i}}\right)\right)_{q}
\end{aligned}
$$




$$
\begin{aligned}
& \leq \exp \left(c \cdot \sum_{i=1}^{s} \frac{1}{P_{i}^{2}}\right) \cdot\left(\prod_{i=1}^{s}\left(1+\frac{d^{l}\left(P_{i}\right)}{P_{i}}\right)\right)_{q} \\
& \leq \exp \left(\frac{c}{2}\right) \cdot\left(\prod_{i=1}^{s}\left(1+\frac{d^{l}\left(P_{i}\right)}{P_{i}}\right)\right)_{q}\left(\text { easy to prove: } \sum_{i=1}^{s} \frac{1}{P_{i}^{2}} \leq \sum_{i=1}^{\infty} \frac{1}{P_{i}^{2}}<0.49<\frac{1}{2}\right) \\
& =\exp \left(\frac{c}{2}\right) \cdot\left(\sum_{1 \leq n \leq q}|\mathrm{u}(\mathrm{n})| \frac{d^{l}(n)}{n}\right)
\end{aligned}
$$

From Lemma 1, it can be seen: when $l=2, c=4.87 \times\left(\frac{l}{\log 2}\right)^{l}$; when $l \geq 3, c=5.71 \times\left(\frac{l}{\log 2}\right)^{l}$. Substitute it into the above formula to get Lemma 2 . The proving process is completed.

Lemma 3: The aim is to prove that:

When $l \geq 2, \sum_{1 \leq n \leq q}|\mathrm{u}(\mathrm{n})| \frac{d^{l}(n)}{n} \geq \exp \left(-(l+0.5) \cdot 2^{l-1}\right) \cdot\left(\sum_{1 \leq n \leq \sqrt{q}}|\mathrm{u}(\mathrm{n})| \frac{d^{l-1}(n)}{n}\right)^{2}$

The proving process is:

$$
\begin{aligned}
& \sum_{1 \leq n \leq q}|\mathrm{u}(\mathrm{n})| \frac{d^{l}(n)}{n}=\sum_{1 \leq n \leq q}\left|\mathrm{u}\left(\mathrm{n}_{1} \mathrm{n}_{2}\right)\right| \frac{d^{l-1}\left(n_{1}\right)}{n_{1}} \sum_{1 \leq n_{2} \leq q / n_{1}} \frac{d^{l-1}\left(n_{2}\right)}{n_{2}} \\
& =\sum_{1 \leq n \leq q}\left|\mathrm{u}\left(\mathrm{n}_{1}\right)\right| \frac{d^{l-1}\left(n_{1}\right)}{n_{1}} \sum_{\substack{1 \leq n_{2} \leq q / n_{1} \\
\left(\mathrm{n}_{1}, n_{2}\right)-1}}\left|\mathrm{u}\left(\mathrm{n}_{2}\right)\right| \frac{d^{l-1}\left(n_{2}\right)}{n_{2}} \\
& \geq \sum_{1 \leq n \leq \sqrt{q}}\left|\mathrm{u}\left(\mathrm{n}_{1}\right)\right| \frac{d^{l-1}\left(n_{1}\right)}{n_{1}} \sum_{\substack{1 \leq n_{2} \leq \sqrt{q} \\
\left(\mathrm{n}_{1}, \mathrm{n}_{2}\right)-1}}\left|\mathrm{u}\left(\mathrm{n}_{2}\right)\right| \frac{d^{l-1}\left(n_{2}\right)}{n_{2}} \\
& \left.=\sum_{1 \leq n \leq \sqrt{q}}\left|\mathrm{u}\left(\mathrm{n}_{1}\right)\right| \frac{d^{l-1}\left(n_{1}\right)}{n_{1}} \cdot \prod_{\substack{1 \leq n_{2} \leq \sqrt{q} \\
\left(\mathrm{n}_{1}, \mathrm{n}_{2}\right)-1}}\left(1+\frac{d^{l-1}\left(P_{i}\right)}{P_{i}}\right)\right)_{\sqrt{q}} \text { Formula }
\end{aligned}
$$

Let the prime number be $P_{\alpha}^{\prime} \mid n_{1}$. It is easy to know $P_{\alpha}^{\prime} \leq n_{1} \leq \sqrt{q}$. If $u\left(n_{1}\right) \neq 0$, then

$$
\begin{aligned}
& \left(\prod_{\substack{1 \leq P_{i} \leq \sqrt{q} \\
\left(\frac{n_{1}}{\left.P_{\alpha}, P_{i}\right)=1}\right.}}\left(1+\frac{d^{l-1}\left(P_{i}\right)}{P_{i}}\right)\right)_{\sqrt{q}}=\left(\prod_{\substack{1 \leq P_{i} \leq \sqrt{q} \\
\left(\mathrm{n}_{1}, P_{i}\right)=1}}\left(1+\frac{d^{l-1}\left(P_{i}\right)}{P_{i}}\right)\right)_{\sqrt{q}}+\frac{d^{l-1}\left(P_{\alpha}^{\prime}\right)}{P_{\alpha}^{\prime}}\left(\prod_{\substack{1 \leq P_{i} \leq \sqrt{q} \\
\left(\mathrm{n}_{1}, P_{i}\right)=1}}\left(1+\frac{d^{l-1}\left(P_{i}\right)}{P_{i}}\right)\right)_{\sqrt{q} / P_{\alpha}^{\prime}} \\
\leq & \left(\prod_{\substack{1 \leq P_{i} \leq \sqrt{q} \\
\left(\mathrm{n}_{1}, P_{i}\right)=1}}\left(1+\frac{d^{l-1}\left(P_{i}\right)}{P_{i}}\right)\right)_{\sqrt{q}}+\frac{d^{l-1}\left(P_{\alpha}^{\prime}\right)}{P_{\alpha}}\left(\prod_{\substack{1 \leq P_{i} \leq \sqrt{q} \\
\left(\mathrm{n}_{1}, P_{i}\right)=1}}\left(1+\frac{d^{l-1}\left(P_{i}\right)}{P_{i}}\right)\right)_{\sqrt{q}} \\
= & \left(1+\frac{d^{l-1}\left(P_{i}\right)}{P_{i}}\right) \cdot\left(\prod_{\substack{1 \leq P_{i} \leq \sqrt{q} \\
\left(\mathrm{n}_{1}, P_{i}\right)=1}}\left(1+\frac{d^{l-1}\left(P_{i}\right)}{P_{i}}\right)\right)_{\sqrt{q}} \\
\Rightarrow & \left(\prod_{\substack{1 \leq P_{i} \leq \sqrt{q} \\
\left(\mathrm{n}_{1}, P_{i}\right)=1}}\left(1+\frac{d^{l-1}\left(P_{i}\right)}{P_{i}}\right)\right)_{\sqrt{q}} \geq\left(1+\frac{d^{l-1}\left(P_{\alpha}^{\prime}\right)}{P_{\alpha}^{\prime}}\right)^{-1}\left(\prod_{\substack{1 \leq P_{i} \leq \sqrt{q} \\
\left(\mathrm{n}_{1}, P_{i}\right)=1}}\left(1+\frac{d^{l-1}\left(P_{i}\right)_{i}}{P_{i}}\right)\right)_{\sqrt{q}}
\end{aligned}
$$

Similarly, set $n_{1}=P_{\alpha 1}{ }^{\prime} P_{\alpha 2}{ }^{\prime} \cdots P_{\alpha m}{ }^{\prime}$, and substitute Formula (2) into Formula (1) to get:

$$
\sum_{1 \leq n \leq \sqrt{q}}|\mathrm{u}(\mathrm{n})| \frac{d^{l-1}(n)}{n} \geq \sum_{\substack{1 \leq n_{n} \leq \sqrt{q} \\ \mathrm{P}_{\alpha}^{\prime} \mid \mathrm{n}_{1}}}\left|\mathrm{u}\left(\mathrm{n}_{1}\right)\right| \frac{d^{l-1}\left(n_{1}\right)}{n_{1}} \cdot\left(1+d^{l-1}\left(\mathrm{P}_{\alpha}^{\prime}\right) \cdot P_{\alpha}^{\prime-1}\right)^{-1}
$$




$$
\begin{aligned}
& \left.\times \prod_{i=1}^{s}\left(1+\frac{d^{l-1}\left(P_{i}\right)}{P_{i}}\right)\right)_{\sqrt{q}} \\
& \left(\frac{n_{1}}{P_{\alpha}}, P_{i}\right)=1 \\
& =\sum_{1 \leq P_{\alpha 1}{ }^{\prime}, P_{\alpha 2}{ }^{\prime} \cdots P_{\alpha m}{ }^{\prime} \leq \sqrt{q}}\left|\mathrm{u}\left(P_{\alpha 1}{ }^{\prime}, P_{\alpha 2}{ }^{\prime} \cdots P_{\alpha m}{ }^{\prime}\right)\right| \cdot \frac{d^{l-1}\left(\frac{n_{1}}{P_{\alpha}^{\prime}}\right)}{\frac{n_{1}}{P_{\alpha}^{\prime}}} \cdot\left(\frac{d^{l-1}\left(P_{\alpha}^{\prime}\right) \cdot\left(1+d^{l-1}\left(P_{\alpha}^{\prime}\right) P_{\alpha}^{\prime-1}\right)^{-1}}{P_{\alpha}^{\prime}}\right)
\end{aligned}
$$

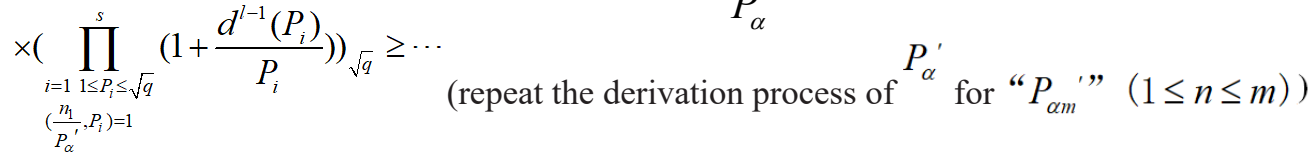

$$
\begin{aligned}
& \geq \sum_{1 \leq P_{\alpha 1}{ }^{\prime}, P_{\alpha 2}{ }^{\prime} \cdots P_{\alpha m}{ }^{\prime} \leq \sqrt{q}}\left|\mathrm{u}\left(P_{\alpha 1}^{\prime}, P_{\alpha 2}{ }^{\prime} \cdots P_{\alpha m}{ }^{\prime}\right)\right| \cdot\left(\prod_{i=1}^{m}\left(\frac{d^{l-1}\left(P_{\alpha}^{\prime}\right) \cdot\left(1+d^{l-1}\left(P_{\alpha}^{\prime}\right) P_{\alpha}^{\prime-1}\right)^{-1}}{P_{\alpha}^{\prime}}\right)\right. \\
& \times\left(\prod_{i=1}^{s}\left(1+\frac{d^{l-1}\left(P_{i}\right)}{P_{i}}\right)\right)_{\sqrt{q}} \\
& \left.=\left(\prod_{\alpha=1}^{s}\left(1+\frac{d^{l-1}\left(P_{\alpha}^{\prime}\right) \cdot\left(1+d^{q}\right.}{P_{\alpha}}\left(P_{\alpha}\right) P_{\alpha}^{-1}\right)^{-1}\right)\right) \underset{\sqrt{q}}{\bullet} \cdot\left(\sum_{1 \leq n \leq \sqrt{q}}|\mathrm{u}(\mathrm{n})| \frac{d^{l-1}(n)}{n}\right) \\
& \left.\geq \prod_{\alpha=s^{\prime}+1}^{s}\left(1+\frac{d^{l-1}\left(P_{\alpha}^{\prime} \leq \sqrt{q}\right) \cdot\left(1+d^{l-1}\left(P_{\alpha}\right) P_{\alpha}^{-1}\right)^{-1}}{P_{\alpha}}\right)\right) \underset{\sqrt{q}}{\bullet}\left(\sum_{1 \leq n \leq \sqrt{q}}|\mathrm{u}(\mathrm{n})| \frac{d^{l-1}(n)}{n}\right) \\
& \text { (Meet condition } \left.\frac{d^{l-1}\left(P_{s^{\prime}+1}\right)}{P_{s^{\prime}+1}}<\frac{1}{\sqrt{2}} \leq \frac{d^{l-1}\left(P_{s^{\prime}}\right)}{P_{s^{\prime}}}\right) \text {, namely, } P_{s^{\prime}} \leq 2^{l-\frac{1}{2}}<P_{s^{\prime}+1} \text { Formula(3) } \\
& \left(\prod_{\alpha=s^{\prime}+1}^{s}\left(1+\frac{d^{l-1}\left(P_{\alpha}\right) \cdot\left(1+d^{l-1}\left(P_{\alpha}\right) P_{\alpha}^{-1}\right)^{-1}}{P_{\alpha}}\right)\right)_{\sqrt{q}}
\end{aligned}
$$

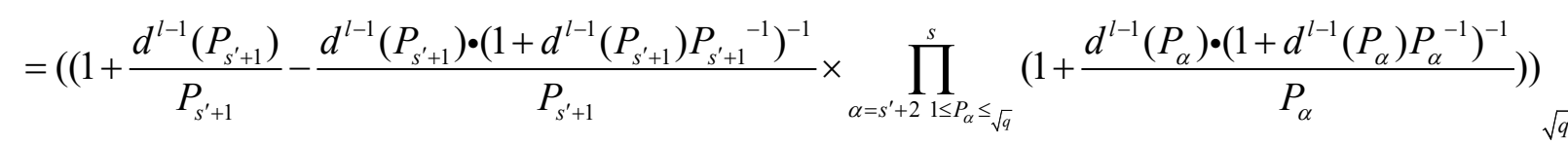

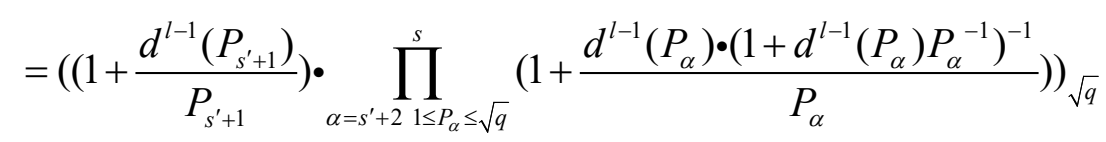

$$
\begin{aligned}
& -\frac{d^{2(1-1)}\left(P_{s^{\prime}+1}\right) \cdot\left(1+d^{l-1}\left(P_{s^{\prime}+1}\right) P_{s^{\prime}+1}^{-1}\right)^{-1}}{P^{2}} \cdot \prod_{\alpha=s^{\prime}+2}^{s}\left(1 \leq P_{\alpha} \leq \sqrt{q}\left(\frac{d^{l-1}\left(P_{\alpha}\right) \cdot\left(1+d^{l-1}\left(P_{\alpha}\right) P_{\alpha}^{-1}\right)^{-1}}{P_{\alpha}}\right)\right)_{\sqrt{q} / P_{s^{\prime}+1}}
\end{aligned}
$$

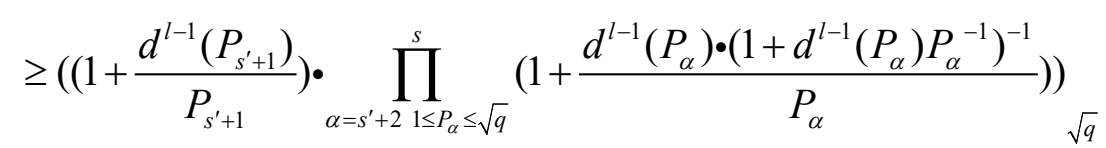

$$
\begin{aligned}
& -\frac{d^{2(q-1)}(P, \quad)}{P,}\left(\prod_{=s^{\prime}+2}\left(1 \leq \frac{d^{l-1}\left(P_{\alpha}\right)\left(1 d^{l-1}\left(P_{\alpha}\right) P_{\alpha}^{-1}\right)^{-1}}{P}\right)\right)
\end{aligned}
$$

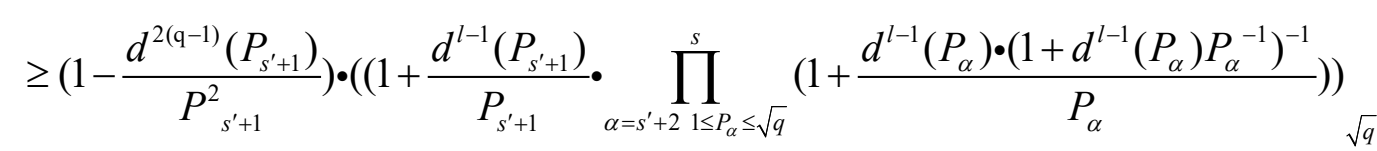


From the condition given by Formula (3), it is easy to know: $1-\frac{d^{2}\left(P_{s^{\prime}+1}\right)}{P_{s^{\prime}+1}^{2}}>0$

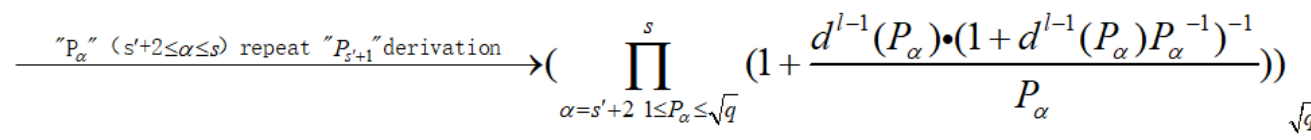

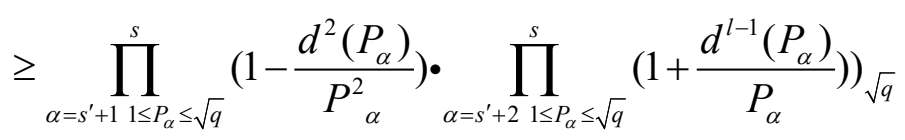

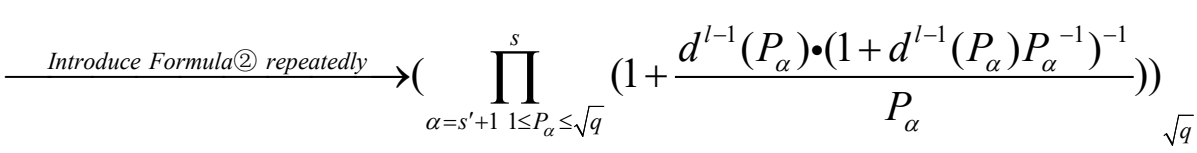

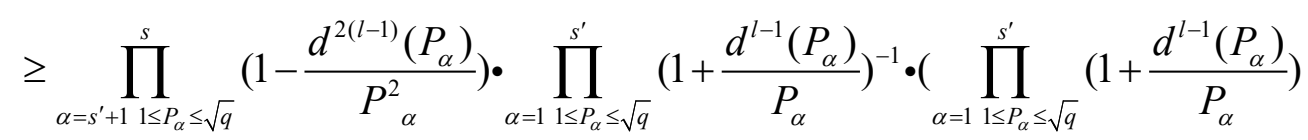

$$
\begin{aligned}
& \text { - } \left.\left.\prod_{\alpha=s^{\prime}+1}^{s}\left(1+\frac{d^{l-1}\left(P_{\alpha}\right)}{P_{\alpha}}\right)\right)_{\sqrt{q}}\right)
\end{aligned}
$$

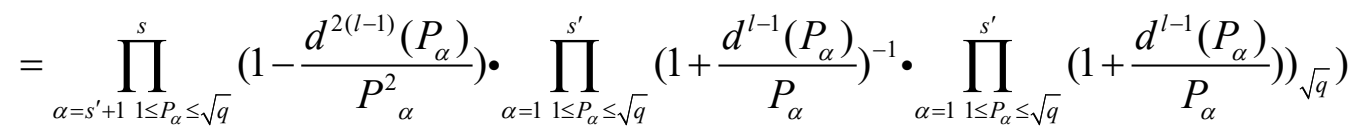

$$
\begin{aligned}
& =\exp \left(-\sum_{\alpha=1}^{s^{\prime}} \log \left(1+\frac{d^{(l-1)}\left(P_{\alpha}\right)}{P_{\alpha}}\right)\right. \\
& \left.+\sum_{\alpha=s^{\prime}+1}^{s} \log \left(1-\frac{d^{2(l-1)}\left(P_{\alpha}\right)}{P_{\alpha}^{2}}\right)\right) \cdot\left(\sum_{1 \leq n \leq \sqrt{q}}|u(n)| \frac{d^{(l-1)}(\mathrm{n})}{n}\right) \\
& \text { When } \frac{d^{(l-1)}\left(P_{\alpha}\right)}{P_{\alpha}}>0, \Rightarrow-\log \left(1+\frac{d^{(l-1)}\left(P_{\alpha}\right)}{P_{\alpha}}\right)>-\frac{d^{(l-1)}\left(P_{\alpha}\right)}{P_{\alpha}} \\
& \text { When } \frac{d^{2(l-1)}\left(P_{\alpha}\right)}{P_{\alpha}^{2}} \leq \frac{1}{2}, \Rightarrow-\log \left(1-\frac{d^{2(l-1)}\left(P_{\alpha}\right)}{P_{\alpha}^{2}}\right)>-2 \frac{d^{2(l-1)}\left(P_{\alpha}\right)}{P_{\alpha}^{2}} \\
& \left.\Rightarrow \prod_{\alpha=s^{\prime}+1}^{s}\left(1+\frac{d^{(l-1)}\left(P_{\alpha} \leq \sqrt{q}\right.}{\left(1+d^{(l-1)}\left(P_{\alpha}\right) P_{\alpha}^{-1}\right)^{-1}}\right)\right)_{\sqrt{q}} \\
& \left.\geq \exp \left(-\sum_{\alpha=1}^{s^{\prime}} \frac{d^{(l-1)}\left(P_{\alpha} \leq \sqrt{q}\right.}{P_{\alpha}}\right)-2 \sum_{\alpha=s^{\prime}+1}^{s} \frac{d^{2(l-1)}\left(P_{\alpha}\right)}{P_{\alpha}^{2}}\right) \cdot\left(\sum_{1 \leq n \leq \sqrt{q}}|u(n)| \frac{d^{(l-1)}(\mathrm{n})}{n}\right) \\
& \geq \exp \left(-2^{l-1} \cdot \log \mathrm{P}_{s^{\prime}}-\frac{2^{2 l-1}}{P_{s^{\prime}+1}}\right) \cdot\left(\sum_{1 \leq n \leq \sqrt{q}}|u(n)| \frac{d^{(l-1)}(\mathrm{n})}{n}\right) \\
& \geq \exp \left(-(l+0.5) \cdot 2^{l-1}\right) \cdot\left(\sum_{1 \leq n \leq \sqrt{q}}|u(n)| \frac{d^{(l-1)}(\mathrm{n})}{n}\right)\left(\text { use } l \geq 2 \text { and } P_{\mathrm{s}^{\prime}} \leq 2^{l-\frac{1}{2}}<P_{\mathrm{s}^{\prime}+1}\right)
\end{aligned}
$$

Substitute the above formula into Formula (3) : 
$\sum_{1 \leq n \leq \sqrt{q}}|u(n)| \frac{d^{l}(\mathrm{n})}{n} \geq \exp \left(-(l+0.5) \cdot 2^{l-1}\right) \cdot\left(\sum_{1 \leq n \leq \sqrt{q}}|u(n)| \frac{d^{(l-1)}(\mathrm{n})}{n}\right)^{2}$

The proving process is completed.

\subsection{Proof of the conclusion}

The proving process is:

1) When $l=3$,

$$
\sum_{1 \leq n \leq q} \frac{d^{3}(\mathrm{n})}{n} \geq \sum_{1 \leq n \leq q}|u(n)| \frac{d^{3}(\mathrm{n})}{n}
$$

$\left.\geq \exp \left(-(3+0.5) \cdot 2^{2}\right) \cdot \sum_{1 \leq n \leq \sqrt{q}}|u(n)| \frac{d^{2}(\mathrm{n})}{n}\right)^{2}$ (gotten from Lemma 3)

$\geq \exp (-14) \cdot\left(\exp \left(-2.44 \times\left(\frac{2}{\log 2}\right)^{2}\right) \cdot\left(\sum_{1 \leq n \leq \sqrt{q}} \frac{d^{2}(\mathrm{n})}{n}\right)^{2}\right.$ (gotten from Lemma 2$)$

$\geq \exp (-55) \cdot\left(\sum_{1 \leq n \leq \sqrt{q}} \frac{d^{2}(\mathrm{n})}{n}\right)^{2}$

From $\sum_{1 \leq n \leq \sqrt{q}} \frac{d^{2}(\mathrm{n})}{n}=\frac{1}{4 \pi^{2}} \log ^{4} q+O\left(\log ^{3} \mathrm{q}\right)$

When q is sufficiently large, $\sum_{1 \leq n \leq \sqrt{q}} \frac{d^{2}(\mathrm{n})}{n}>\frac{1}{40} \log ^{4} q$

$\Rightarrow \sum_{1 \leq n \leq \sqrt{q}} \frac{d^{3}(\mathrm{n})}{n} \geq \exp (-55) \cdot\left(\frac{1}{40} \log ^{4} \sqrt{q}\right)^{2}$

$\geq \exp (-68) \log ^{8} q>\exp \left(-0.84 \cdot\left(\frac{l}{\log 2}\right)^{l}\right) \cdot \log ^{2^{l}} q$ (It makes sense when $l=3$.)

That is: when $l=3, \quad l=3, \sum_{1 \leq n \leq q} \frac{d^{l}(\mathrm{n})}{n} \geq \exp \left(-0.84 \cdot\left(\frac{l}{\log 2}\right)^{l}\right) \cdot \log ^{2^{l}} q$ makes sense.

2) When $l \geq 4$,

If $l=4, \sum_{1 \leq n \leq q} \frac{d^{4}(\mathrm{n})}{n} \geq \sum_{1 \leq n \leq q}|u(n)| \frac{d^{4}(\mathrm{n})}{n}$

$\geq \exp \left(-(4+0.5) \cdot 2^{3}\right) \cdot\left(\sum_{1 \leq n \leq \sqrt{q}}|u(n)| \frac{d^{3}(\mathrm{n})}{n}\right)^{2} \quad$ gotten from Lemma 3$)$

$\geq \exp (-36) \cdot\left(\exp \left(-2.86 \times\left(\frac{3}{\log 2}\right)^{3}\right) \cdot\left(\sum_{1 \leq n \leq \sqrt{q}} \frac{d^{3}(\mathrm{n})}{n}\right)^{2}\right.$ (gotten from Lemma 2)

$\geq \exp (-500) \cdot\left(\sum_{1 \leq n \leq \sqrt{q}} \frac{d^{3}(\mathrm{n})}{n}\right)^{2}$

$\geq \exp (-500) \cdot\left(\exp (-68) \cdot \log ^{8} \sqrt{q}\right)^{2}$ (the result calculated when quoting $l=3$ directly)

$\geq \exp (-648) \cdot \log ^{2^{4}} q \geq \exp \left(-0.59 \cdot\left(\frac{l}{\log 2}\right)^{l}\right) \cdot \log ^{2^{l^{l}}} q($ It makes sense when $l=4$.) 
That is: when $l=4, \sum_{1 \leq n \leq q} \frac{d^{l}(\mathrm{n})}{n} \geq \exp \left(-0.59 \cdot\left(\frac{l}{\log 2}\right)^{l}\right) \cdot \log ^{2^{l}} q$

If when $l=k, \sum_{1 \leq n \leq q} \frac{d^{l}(\mathrm{n})}{n} \geq \exp \left(-0.59 \cdot\left(\frac{l}{\log 2}\right)^{l}\right) \cdot \log ^{2^{l}} q$ also makes sense,

then when $l=k+1, \sum_{1 \leq n \leq q} \frac{d^{k+1}(\mathrm{n})}{n} \geq \sum_{1 \leq n \leq q}|u(n)| \frac{d^{k+1}(\mathrm{n})}{n}$

$\geq \exp \left(-(\mathrm{k}+1+0.5) \cdot 2^{k}\right) \cdot\left(\sum_{1 \leq n \leq \sqrt{q}}|u(n)| \frac{d^{k}(\mathrm{n})}{n}\right)^{2}$ (gotten from Lemma 3)

$\geq \exp \left((-k+1.5) \cdot 2^{k}\right) \cdot\left(\exp \left(-2.86 \times\left(\frac{k}{\log 2}\right)^{k}\right) \cdot \sum_{1 \leq n \leq \sqrt{q}} \frac{d^{k}(\mathrm{n})}{n}\right)^{2}$ (gotten from Lemma 2)

$\geq \exp \left((-k+1.5) \cdot 2^{k}\right) \cdot\left(\exp \left(-2.86 \times\left(\frac{k}{\log 2}\right)^{k}\right)\right.$

$\cdot \exp \left(-0.59 \times\left(\frac{k}{\log 2}\right)^{k} \cdot \log ^{2^{k}} \sqrt{q}\right)^{2}$ (assuming that it also holds when $l=k$ )

$=\exp \left((-k+1.5) \cdot 2^{k}-6.9 \cdot\left(\frac{k}{\log 2}\right)^{k}-2^{k+1} \log 2\right) \cdot \log 2^{k+1} q$

$\geq \exp \left(-0.59 \times\left(\frac{k+1}{\log 2}\right)^{k+1} \cdot \log ^{2^{k+1}} q(\right.$ assuming that it also holds when $l \geq 4)$

That is: when $l \geq 4, \sum_{1 \leq n \leq q} \frac{d^{l}(\mathrm{n})}{n} \geq \exp \left(-0.59 \cdot\left(\frac{l}{\log 2}\right)^{l}\right) \cdot \log ^{2^{l}} q$ makes sense.

3) When $l=2$, it can be known from $\sum_{1 \leq n \leq q} \frac{d^{2}(\mathrm{n})}{n}=\frac{1}{4 \pi^{2}} \log ^{4} q+O\left(\log ^{3} \mathrm{q}\right)$ that when $q$ is sufficiently large, $\sum_{1 \leq n \leq q} \frac{d^{2}(\mathrm{n})}{n} \leq(\exp (-2 \cdot \log 2 \pi)+\varepsilon) \cdot \log ^{4} q(\varepsilon>0)$ holds.

Assume that when $l=k, \sum_{1 \leq n \leq q} \frac{d^{k}(\mathrm{n})}{n} \leq\left(\exp \left(-2^{k-1} \cdot \log 2 \pi\right)+\varepsilon\right) \cdot \log ^{2^{k}} q(\varepsilon>0)$ holds. Then when $l=\mathrm{k}+1$ ,$\sum_{1 \leq n \leq q} \frac{d^{k+1}(\mathrm{n})}{n} \leq \sum_{1 \leq n_{1} \leq q} \frac{d^{k}\left(\mathrm{n}_{1}\right)}{n_{1}} \sum_{1 \leq n_{2} \leq q / n_{1}} \frac{d^{k}\left(\mathrm{n}_{2}\right)}{n_{2}}\left(\right.$ use $\left.d\left(n_{1}\right) d\left(n_{2}\right) \geq d\left(n_{1} n_{2}\right)\right)$

$\leq \sum_{1 \leq n_{1} \leq q} \frac{d^{k}\left(\mathrm{n}_{1}\right)}{n_{1}} \sum_{1 \leq n_{2} \leq q} \frac{d^{k}\left(\mathrm{n}_{2}\right)}{n_{2}}$

$\leq\left\{\left(\exp \left(-2^{k-1} \cdot \log 2 \pi+\varepsilon\right) \cdot \log ^{2^{k}} q\right\}^{2}\right.$ (assuming that it also holds when $\left.l=k\right)$

$\leq\left(\exp \left(-2^{k} \cdot \log 2 \pi\right)+\varepsilon^{\prime}\right) \cdot \log ^{2^{k+1}} q\left(\right.$ when $\varepsilon$ is small enough, $\varepsilon^{\prime} \leq \varepsilon$ is easy to be proved $)$

That is: when $q$ is sufficiently large, if $l \geq 2$,

$$
\sum_{1 \leq n \leq q} \frac{d^{k}(\mathrm{n})}{n} \leq\left(\exp \left(-2^{k-1} \cdot \log 2 \pi\right)+\varepsilon\right) \cdot \log ^{2^{k}} q(\varepsilon>0) \text { makes sense. }
$$

Based on the conclusions obtained above, it can be seen that the theorem makes sense. And the proving process is completed.

Note: "Theorem 1" actually proves " $f_{1}(l) \log ^{2^{l}} \mathrm{q}<\sum_{1 \leq n \leq q} \frac{d^{k}(\mathrm{n})}{n} f_{2}(l) \log ^{2^{l}} \mathrm{q}$ ”, but as far as the form of " $f_{1}(l)$ " and " $f_{2}(l)$ " is concerned, there is obviously still much room for improvement. Due to limited space, no further research will be done here. 


\section{Research on the solution number of the polynomial with integer coefficients " $g(x) \equiv 0(\operatorname{modq})$ "}

The author once wanted to improve the Theorem 1 in Additive Theory of Prime Numbers by studying the solution number of " $g(x) \equiv 0$ (modq)", which is currently unsatisfactory, but some of the deduced conclusions help to improve Theorem 3 related to "divisor function" in Additive Theory of Prime Numbers. This article will discuss this part ${ }^{[2]}$.

\subsection{The conclusion to be proved}

Theorem 2: If let $\mathrm{g}(x)=b_{k} x^{k}+b_{k-1} x^{k-1}+\cdots+b_{1} x+b_{\text {satisfy the condition }}\left(b_{k}, b_{k-1}, \cdots b_{0}, \mathrm{q}\right)=1$, then the number of solutions of " $g(x) \equiv 0(\operatorname{modq}) " \leq 2^{\pi(2 \mathrm{k})} \cdot q^{1-\frac{1}{k}}$.

\subsection{The proving process of the conclusion-the first half}

Let $P^{m} \| q$ and $P$ be prime numbers, $m \geq 1$

1) First factorize $g(x)$ :

$$
g(x)=\left(x-b_{1}\right)\left(x-b_{2}\right) \cdots\left(x-b_{n}\right) g_{0}(x)
$$

Among them, $b_{i}$ is an integer, and for any integer $x, g_{0}(x) \neq 0$, if the order of $b_{i}$ is not considered, this kind of decomposition is obviously unique.

2) If there is an integer $b_{n+1}$ to make $P^{m} \| g_{0}\left(b_{n+1}\right)$, then $g_{0}(x)=\left(x-b_{n+1}\right) g_{1}(x)+g_{0}\left(b_{n+1}\right)$. It is easy to know that $g_{1}(x)$ is also an polynomial with integer coefficients.

That is, $g(x)=\left(x+b_{1}\right)\left(x-b_{2}\right) \cdots\left(x-b_{n}\right)\left[\left(x-b_{n+1}\right) g_{1}(x)+g_{0}\left(b_{n+1}\right)\right]$

$$
\equiv\left(x-b_{1}\right)\left(x-b_{2}\right) \cdots\left(x-b_{n}\right)\left(x-b_{n+1}\right) g_{1}(x)\left(\operatorname{modP}^{m}\right)
$$

Set $b_{n+1}, b_{n+2}, \cdots, b_{n+n_{0}}$ to satisfy $P^{m} \mid g_{i}\left(b_{n+i}\right)\left(1 \leq i \leq n_{0}\right)$. And it is easy to know from the above derivation: $g(x) \equiv\left(x-b_{1}\right)\left(x-b_{2}\right) \cdots\left(x-b_{n}\right)\left(x-b_{n+1}\right) \cdots\left(x-b_{n+n_{0}}\right) g_{n_{0}}(x)\left(\operatorname{modP}^{m}\right)$. Among them, $g_{n_{0}}(x)$ is available for any integer $x$ until $g_{n_{0}}(x) \neq 0\left(\operatorname{modP}^{m}\right)$.

3) Let $r$ be the maximum value that satisfies the condition " $P^{m} \mid g_{n_{0}}(x)$ " ${ }_{r} \mathbf{r} 1^{\text {and }}$ is an integer). That is, " $P^{r+1} \mid g_{n_{0}}(x)$ " is not true for any integer $x$. But there must be an integer $x$, so that " $P^{r} \mid g_{n_{0}}(x)$ " holds. Assume that " $P^{r} \mid g_{n_{0}}\left(b_{n+n_{0}+1}\right)$ " makes sense, and then $g_{n_{0}}(x)=\left(x-b_{n+n_{0}+1}\right) g_{n_{0}+1}(x)+g_{n_{0}}\left(b_{n+n_{0}+1}\right)$. For any integer $x_{0}$, there is $P^{r_{1}}\left\|\left(x_{0}-b_{n+n_{0}+1}\right) g_{n_{0}+1}\left(x_{0}\right), P^{r_{2}}\right\| g_{n_{0}}\left(x_{0}\right)$. It is easy to know from the previous assumption: when $\mathrm{r}_{1} \geq r$, $r_{2}=r \leq \mathrm{r}_{1}$; when $r_{1}<r, r_{1}=r_{2}<r$, namely: $r_{2} \leq \mathrm{r}_{1}$, or $P^{r_{2}} \| P^{r_{1}}$. Now

$g_{1}(x)=\left(x-b_{1}\right)\left(x-b_{2}\right) \cdots\left(x-b_{n+n_{0}}\right)\left(x-b_{n+n_{0}+1}\right) g_{n_{0}+1}(x)$, and then: the solution number of $g_{1}(x) \equiv 0\left(\operatorname{modP}^{m}\right)$ must not be less than the solution number of $g(x) \equiv 0\left(\operatorname{modP} \mathrm{P}^{m}\right)$.

Repeat the above process until $g_{n_{0}^{\prime}}\left(x_{0}\right) \not \equiv 0(\operatorname{modP})$ for any integer $x_{0}$.

Note: $g_{1}^{\prime}(x)=\left(x-b_{1}\right)\left(x-b_{2}\right) \cdots\left(x-b_{n+n_{0}}\right)\left(x-b_{n+n_{0}+1}\right)\left(x-b_{k^{\prime}}\right) g_{n_{0}}{ }^{\prime}(x) \equiv 0\left(\operatorname{modP} P^{m}\right)$, and then $f(x)=\left(x-b_{1}\right) \cdots\left(x-b_{n+n_{0}}\right)\left(x-b_{n+n_{0}+1}\right) \cdots\left(x-b_{k^{\prime}}\right) g_{n_{0}}{ }^{\prime}(x) \equiv 0(\operatorname{modP})^{m}$ and $\left.g_{1}^{\prime}(x) \equiv 0(\operatorname{modP})^{m}\right)$ have the same number of solutions. From the previous derivation, it is easy to know: $k^{\prime} \leq k$, but the solution number of $\left.f(x) \equiv 0(\operatorname{modP})^{m}\right)$ is not less than the solution number of $\left.g(x) \equiv 0(\operatorname{modP})^{m}\right)^{[3]}$. 
That is, there is a special function of the form $f(x)=\prod_{i=1}^{k^{\prime}}\left(x-b_{i}\right)\left(k^{\prime} \leq k\right)$, which satisfies the condition that the solution number of $\left.f(x) \equiv 0(\operatorname{modP})^{m}\right)$ is not less than the solution number of $g(x) \equiv 0\left(\operatorname{modP}^{m}\right)$.

\subsection{Proof of several lemmas}

Lemma 1: Let $k_{1}, k_{2}, \cdots, k_{1}$ be positive integers and satisfy the condition $\sum_{i=1}^{l} k_{i}=k^{\prime}$. And then: when $\mathrm{P}^{m} \geq 2^{k^{\prime}}$, $\sum_{i=1}^{l} \mathrm{P}^{m}{ }^{\left(1-\frac{1}{k_{i}}\right)} \leq \mathrm{P}^{m}{ }^{\left(1-\frac{1}{k_{i}}\right)}$

The proving process is:

(1) First prove a proposition. Let $k_{1}, k_{2}$ be positive numbers, and $Q>1$.

If $2^{k_{1}+k_{2}} \leq Q^{\text {, then }} Q^{-\frac{1}{k_{1}}}+Q^{-\frac{1}{k_{2}}} \leq Q^{-\frac{1}{k_{1}+k_{2}}}$.

Make $k_{1}+k_{2}=k_{0}$, and then $f\left(k_{1}\right)=Q^{-\frac{1}{k_{1}}}+Q^{-\frac{1}{k_{2}}}=Q^{-\frac{1}{k_{1}}}+Q^{-\frac{1}{k_{0}-k_{1}}}$.

Because of the symmetry of $k_{1}, k_{2}$ in $f\left(k_{1}\right)$, let $0<k_{1} \leq \frac{k_{0}}{2}$.

Then $f^{\prime}\left(k_{1}\right)=\left(\frac{1}{k_{1}^{2} Q^{\frac{1}{k_{1}}}}-\frac{1}{\left(k_{0}-k_{1}\right)^{2} Q^{\frac{1}{k_{0}-k_{1}}}}\right) \log \mathrm{Q}=\left(\mathrm{g}^{-1}\left(\mathrm{k}_{1}\right)-\mathrm{g}^{-1}\left(\mathrm{k}_{0}-\mathrm{k}_{1}\right)\right) \log \mathrm{Q}$.

Among them, $\mathrm{g}\left(k_{1}\right)=\mathrm{k}_{1}^{2} Q^{\frac{1}{k_{1}}}$.

It can be known from $\mathrm{g}^{\prime}\left(k_{1}\right)=\left(2 \mathrm{k}_{1}-\log \mathrm{Q}\right) Q^{\frac{1}{k_{1}}}$ that when $2 \mathrm{k}_{1}>\log \mathrm{Q}{ }^{\prime} \mathrm{g}^{\prime}\left(k_{1}\right)>0 \cdot g\left(k_{1}\right)$ is an increasing function and $g^{-1}\left(k_{1}\right)$ is a decreasing function. $g^{-1}\left(k_{1}\right)-g^{-1}\left(\mathrm{k}_{0}-k_{1}\right)>0$ can be known from $k_{1} \in\left[0, \frac{k_{0}}{2}\right]$ (when $\left.k_{1} \in\left[0, \frac{k_{0}}{2}\right]\right) . \log Q>0$ can be known from $Q>1$. That is $f^{\prime}\left(k_{1}\right)>0$, and $f\left(k_{1}\right)$ is an increasing function. Similarly, it can be proved that $2 \mathrm{k}_{1}>\log \mathrm{Q}$, and that $f\left(k_{1}\right)$ is a decreasing function when $k_{1} \in\left[0, \frac{k_{0}}{2}\right]$. That is, the maximum value of $f\left(k_{1}\right)$ can only be obtained at the endpoint $f\left(0^{+}\right), f\left(\frac{k_{0}}{2}\right)$ or $f^{\prime}\left(k_{0}\right)=0$.

Because $k_{1}=\frac{k_{0}}{2}$ when $f^{\prime}\left(k_{0}\right)=0$, so the maximum value of $f\left(k_{1}\right)$ can be obtained as long as the size of the two endpoints of $f\left(0^{+}\right)$and $f\left(\frac{k_{0}}{2}\right)$. If $f\left(0^{+}\right) \geq f\left(\frac{k_{0}}{2}\right)$, then $Q^{-\frac{1}{k_{1}}} \geq 2 Q^{-\frac{2}{k_{0}}}$, which means $Q \geq 2^{k_{0}}$. This proves: when $Q \geq 2^{k_{1}+k_{2}}, Q^{-\frac{1}{k_{1}}}+Q^{-\frac{1}{k_{2}}} \leq f\left(0^{+}\right)=Q^{-\frac{1}{k_{1}+k_{2}}}$. Take $Q=P^{m}$, and

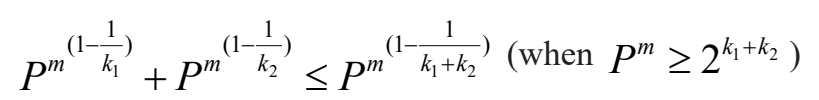

(2) Prove $\sum_{i=1}^{l} P^{m^{\left(1-\frac{1}{k_{i}}\right)}} \leq P^{m^{\left(1-\frac{1}{k^{\prime}}\right)}}$ next.

When $l=1, \sum_{i=1}^{l} P^{m^{\left(1-\frac{1}{k_{i}}\right)}} \leq P^{m^{\left(1-\frac{1}{k^{\prime}}\right)}}$ clearly makes sense. 
When $l \geq 2, \sum_{i=1}^{l} P^{m^{\left(1-\frac{1}{k_{i}}\right.}}=P^{m^{\left(1-\frac{1}{k_{1}}\right)}}+P^{m^{\left(1-\frac{1}{k_{2}}\right)}}+\sum_{i=3}^{l} P^{m^{\left(1-\frac{1}{k_{i}}\right)}}$

It's easy to know from $k_{i} \geq 1: P^{m} \geq 2^{k^{\prime}} \geq 2^{k_{1}+k_{2}}$

$\Rightarrow \sum_{i=1}^{l} P^{m^{\left(1-\frac{1}{k_{i}}\right)}} \leq P^{m^{\left(1-\frac{1}{k_{1}+k_{2}}\right)}}+\sum_{i=2}^{l} P^{m^{\left(1-\frac{1}{k_{i}}\right)}}$

$=P^{m^{\left(1-\frac{1}{k_{1}+k_{2}}\right)}}+P^{m^{\left(1-\frac{1}{k_{3}}\right)}}+\sum_{i=4}^{l} P^{m^{\left(1-\frac{1}{k_{i}}\right)}}$

$\leq P^{m^{\left(1-\frac{1}{k_{1}+k_{2}+k_{3}}\right.}}+\sum_{i=4}^{l} P^{m^{\left(1-\frac{1}{k_{i}}\right)}}$

$\leq \cdots \leq P^{m^{\left(1-\frac{1}{k^{\prime}}\right)}}$

The proving process is completed.

Lemma 2: When $P^{m} \leq 2^{k^{\prime}}$, the number of solutions of $f(x) \equiv 0\left(\bmod P^{m}\right)$

$\leq \min \left\{2 k^{\prime} P^{-1}, 2\right\} \cdot P^{m^{\left(1-\frac{1}{k^{\prime}}\right)}}\left(\right.$ where $\left.f(x)=\prod_{i=1}^{k^{\prime}}\left(x-b_{i}\right)\right)$

The proving process is: the solution number of $f(x) \equiv 0\left(\bmod P^{m}\right) \leq \min \left\{k^{\prime} P^{m-1}, P^{m}\right\}$

$\leq \min \left\{k^{\prime} P^{\frac{m}{k^{\prime}}-1}, P^{\frac{m}{k^{\prime}}}\right\} \cdot P^{m^{\left(1-\frac{1}{k^{\prime}}\right)}}$ and $P^{m} \leq 2^{k^{\prime}} \Rightarrow P^{\frac{m}{k^{\prime}}} \leq 2$

$\Rightarrow$ The solution number of $\Rightarrow f(x) \equiv 0\left(\bmod P^{m}\right) \leq \min \left\{2 k^{\prime} P^{-1}, 2\right\} \cdot P^{m^{\left(1-\frac{1}{k^{\prime}}\right)}}$

The proving process is completed.

Lemma 3: The aim is to prove when $P^{m} \leq 2^{k^{\prime}}$, the solution number of $f(x) \equiv 0\left(\bmod P^{m}\right)$

$\leq \max \left\{\min \left\{2 k^{\prime} P^{-1}, 2\right\}, 1\right\} \cdot P^{m^{\left(1-\frac{1}{k^{\prime}}\right)}}\left(\right.$ where $f(x)=\prod_{i=1}^{k^{\prime}}\left(x-b_{i}\right)$ )

The proving process is: When $P^{m} \geq 2^{k^{\prime}}$, let $g_{0}(x)=\left(x-b_{1}\right)\left(x-b_{2}\right) \cdots\left(x-b_{k^{\prime}}\right)\left(\right.$ where $\left.k^{\prime \prime} \in\left[1, k^{\prime}\right]\right)$

1) When $\mathrm{m}=1$, the solution number of $g_{0}(x) \equiv 0\left(\bmod P^{m}\right) \leq \min \left\{k^{\prime \prime}, P\right\} \leq k^{\prime \prime}$

(1) If $k^{\prime \prime}=1$, the solution number of $g_{0}(x) \equiv 0\left(\bmod P^{m}\right) 1 \leq \max \left\{\min \left\{2 k^{\prime} P^{-1}, 2\right\}, 1\right\} \cdot P^{m^{\left(1-\frac{1}{k^{\prime}}\right)}}$ will make sense.

(2) If $k^{\prime \prime} \geq 2, k^{\prime \prime} \leq 2^{k^{\prime \prime}-1}$

$$
k^{\prime \prime} \leq k^{\prime} \Rightarrow 2^{k^{\prime \prime}} \leq 2^{k^{\prime}} \leq P^{m} \quad \Rightarrow k^{\prime \prime} \leq P^{m^{\left(1-\frac{1}{k^{\prime}}\right)}}
$$

$\leq \max \left\{\min \left\{2 k^{\prime} P^{-1}, 2\right\}, 1\right\} \cdot P^{m^{\left(1-\frac{1}{k^{\prime}}\right)}}$ will make sense.

It can be known based on (1) and (2) that when $m=1$, the solution number of $g_{0}(x) \equiv 0\left(\bmod P^{m}\right)$ 
$\leq \max \left\{\min \left\{2 k^{\prime} P^{-1}, 2\right\}, 1\right\} \cdot P^{m^{\left(1-\frac{1}{k^{\prime}}\right)}}$ makes sense.

2) Assuming when $m \leq m_{0}-1$, the solution number of $g_{0}(x) \equiv 0\left(\bmod P^{m}\right)$

$\leq \max \left\{\min \left\{2 k^{\prime} P^{-1}, 2\right\}, 1\right\} \cdot P^{m^{\left(1-\frac{1}{k^{\prime}}\right)}}$ makes sense.

The following proves that the conclusion is also true when $m=m_{0}$.

(1) When $k^{\prime \prime}=1$, it makes sense that the solution number of $g_{0}(x) \equiv 0\left(\bmod P^{m}\right)$ is $1 \leq \max \left\{\min \left\{2 k^{\prime \prime} P^{-1}, 2\right\}, 1\right\} \cdot P^{m^{\left(1-\frac{1}{k^{\prime}}\right)}}$. When $k^{\prime \prime} \geq 2$, we discuss in two cases: $2^{k^{\prime \prime}} \geq P^{m_{0}}$ and $2^{k^{\prime \prime}}<P^{m_{0}}$.

(2) When $2^{k^{\prime \prime}} \geq P^{m_{0}}$, the solution number of $g_{0}(x) \equiv 0\left(\bmod P^{m}\right) \leq \max \left\{\min \left\{2 k^{\prime \prime} P^{-1}, 2\right\}, 1\right\} \cdot P^{m^{\left(1-\frac{1}{k^{\prime}}\right.}}$ makes sense, which is easy to know by Lemma $2^{[4]}$.

(3) When $2^{k^{\prime \prime}}<P^{m_{0}}$, first classify the " $x-b_{i}$ " in $g_{0}(x)=\left(x-b_{1}\right)\left(x-b_{2}\right) \cdots\left(x-b_{k^{\prime \prime}}\right)$ according to whether " $x-b_{i}$ " satisfies the condition " $b_{i} \equiv b_{\alpha}(\bmod P)$ ". All items satisfying the condition " $b_{i} \equiv b_{\alpha}(\bmod P)$ " in $g_{0}(x)$ are classified into the same category and denoted as $g_{\alpha}(x)=\left(x-b_{\alpha_{1}}\right)\left(x-b_{\alpha_{2}}\right) \cdots\left(x-b_{\alpha_{k^{\prime \prime}}}\right)$. If $b_{i}$ and other items in a certain item " $x-b_{i}$ " do not satisfy the condition " $b_{i} \equiv b_{\alpha}(\bmod P)$ ", separate them into one category, " $g_{\alpha}(x)=\left(x-b_{\alpha}\right)$ ". If the order is not considered, the only division of $g_{0}(x)$ is $g_{0}(x)=\prod_{i=1}^{l} g_{i}(x)$. It's easy to know that the solution number of $g_{0}(x) \equiv 0\left(\bmod P^{m}\right)$ is equal to the sum of the solution number of each item in “ $g_{i}(x) \equiv 0\left(\bmod P^{m_{0}}\right)(1 \leq i \leq l) "$.

Next study the solution number of $g_{i}(x)=\left(x-b_{i 1}\right)\left(x-b_{i 2}\right) \cdots\left(x-b_{i k 1}\right) \equiv 0\left(\bmod P^{m_{0}}\right)$.

When $k_{i}=1$, the solution number of $g_{i}(x) \equiv 0\left(\bmod P^{m_{0}}\right)$ is 1 .

$\leq \max \left\{\min \left\{2 k_{i} P^{-1}, 2\right\}, 1\right\} \cdot P^{m_{0}^{\left(1-\frac{1}{k^{\prime}}\right)}} \quad$ Formula (1)

When $k_{i} \geq 2$, from $b_{i 1} \equiv b_{i \alpha}(\bmod P)\left(\alpha \in\left[2, \mathrm{k}_{i}\right]\right)$, it's easy to know:

any solution to $g_{i}(x) \equiv 0\left(\bmod P^{m_{0}}\right)$ can be expressed in the form of " $x=P m_{1}+b_{i 1}$ " ( $m_{1}$ is an integer $)$.

That is: if " $x=P m_{1}+b_{i 1}$ " is set without loss of generality, it can be obtained by substituting it into " $g_{i}(x)$ " ${ }^{[5]}$.

$g_{i}(x)=g_{i}\left(P m_{1}+b_{i 1}\right)=P^{k_{i}} m_{1}\left(\mathrm{~m}_{1}-\frac{b_{i 2}-b_{i 1}}{P}\right) \cdots\left(\mathrm{m}_{2}-\frac{b_{k i}-b_{i 1}}{P}\right)=\mathrm{g}_{i}^{0}\left(\mathrm{~m}_{1}\right) \equiv 0\left(\operatorname{modp}^{m_{0}}\right)$. Obvious1y, “ $\frac{b_{i \alpha}-b_{i 1}}{P} "\left(\alpha \in\left[2, \mathrm{k}_{i}\right]\right)$ is an integer.

When $k_{i} \geq m_{0}$, the solution number of $g_{i}(x) \equiv 0\left(\bmod P^{m_{0}}\right)$ is ${ }^{[6]}$ :

$P^{m_{0}-1}=P^{m_{0}\left(1-\frac{1}{m_{0}}\right)} \leq P^{m_{0}{ }^{\left(1-\frac{1}{k_{i}}\right)}} \leq \max \left\{\min \left\{2 k^{\prime \prime} P^{-1}, 2\right\}, 1\right\} \cdot P^{m^{\left(1-\frac{1}{k^{\prime}}\right)}}$ Formula (2)

When $\quad k<m, \quad 1 \leq m_{0}-k_{i} \leq m_{0}-1,1 \leq k_{i} \leq k^{\prime \prime} \leq k^{\prime}, \quad$ namely, $\quad k_{i} \in\left[1, \mathrm{k}^{\prime}\right] . \quad$ It can be 
known from the previous assumption that the solution number of $P^{-k i} g_{i}^{0}\left(\mathrm{~m}_{1}\right) \equiv 0\left(\bmod P^{m_{0}-k_{i}}\right)$ $\leq \max \left\{\min \left\{2 k_{i} P^{-1}, 2\right\}, 1\right\} \cdot P^{\left(m_{0}-k_{i}\right)\left(1-\frac{1}{k^{\prime}}\right)}$. Thus it is easy to know the solution number of $g_{i}(\mathrm{x}) \equiv 0\left(\bmod P^{m}\right)$ $\leq \max \left\{\min \left\{2 k_{i} P^{-1}, 2\right\}, 1\right\} \cdot P^{\left(m_{0}-k_{i}\right)\left(1-\frac{1}{k^{\prime}}\right)} \times P^{k_{i}-1}$ $=\max \left\{\min \left\{2 k_{i} P^{-1}, 2\right\}, 1\right\} \cdot P^{m_{0}\left(1-\frac{1}{k_{i}}\right)}$ Formula (3)

Based on Formula (1), (2) and (3), it can be known that the solution number of $g_{i}(x) \equiv 0\left(\bmod P^{m_{0}}\right)$

$\leq \max \left\{\min \left\{2 k_{i} P^{-1}, 2\right\}, 1\right\} \cdot P^{m_{0}\left(1-\frac{1}{k_{i}}\right)}$ Formula (4)

If the number of solutions of " $g_{i}(x) \equiv 0\left(\bmod P^{m_{0}}\right)$ " is $N_{i}$, the number of solutions of $g_{0}(x) \equiv 0\left(\bmod P^{m_{0}}\right)$ can be easily seen from the previous division: $\sum_{i=1}^{l} N_{i} \leq \sum_{i=1}^{l} \max \left\{\min \left\{2 k_{i} P^{-1}, 2\right\}, 1\right\} \bullet P^{m_{0}\left(1-\frac{1}{k_{i}}\right)}$ (gotten from Formula (4) )

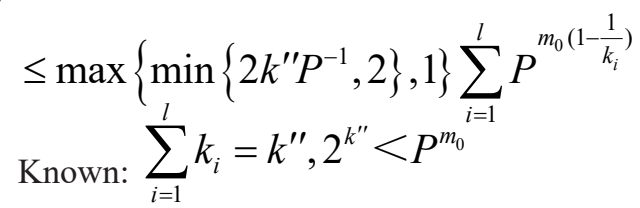

Lemma 1

$\Rightarrow \sum_{i=1}^{l} N_{i} \leq \max \left\{\min \left\{2 k^{\prime \prime} P^{-1}, 2\right\}, 1\right\} \cdot P^{m_{0}\left(1-\frac{1}{k_{i}}\right)}$

It can be seen from the conclusions of (1) (2) (3) in 2): when $\mathrm{m}=\mathrm{m}_{0}$, the solution number of $g_{0}(x) \equiv 0\left(\bmod P^{m_{0}}\right) \leq \max \left\{\min \left\{2 k^{\prime \prime} P^{-1}, 2\right\}, 1\right\} \cdot P^{m_{0}\left(1-\frac{1}{k^{\prime}}\right)}$ also holds.

That is: when $P^{m} \geq 2^{k^{\prime}}$, the solution number of $g_{0}(x) \equiv 0\left(\bmod P^{m_{0}}\right) \leq \max \left\{\min \left\{2 k^{\prime \prime} P^{-1}, 2\right\}, 1\right\} \cdot P^{m_{0}\left(1-\frac{1}{k^{\prime}}\right)}$ holds. Because $k^{\prime \prime} \in\left[1, \mathrm{k}^{\prime}\right], \mathrm{k}^{\prime \prime}=\mathrm{k}^{\prime}$ can be taken (namely, when $g(x)=f(x)$ ). It can be known that the solution number of $f(x) \equiv 0\left(\bmod P^{m_{0}}\right) \leq \max \left\{\min \left\{2 k^{\prime} P^{-1}, 2\right\}, 1\right\} \bullet P^{m_{0}\left(1-\frac{1}{k^{\prime}}\right)}$ holds. The proving process is completed.

\section{Improvement of some conclusions in Chapter 2 of additive theory of prime numbers}

The second part of this article has studied Lemma 2.4 and Lemma 2.5 in Additive Theory of Prime Numbers. This part will study Lemma 2.2, Lemma 2.3 and Theorem 3

The original text is as follows.

Lemma 2.2: Make $f\left(x_{1}, \cdots, x_{n}\right)$ be a polynomial of degree $k$ with integer coefficients, and assume that not all of its coefficients are multiples of $P$. Then ${ }^{[7]}$ :

The solution number of $f\left(x_{1}, \cdots, x_{n}\right) \equiv 0\left(\bmod P^{\alpha}\right) \leq c_{4}(k, n) P^{n \alpha}$

Lemma 2.3: Under the assumption of Lemma 2.2 , the solution number of $f\left(x_{1}, \cdots, x_{n}\right) \equiv 0\left(\bmod P^{\alpha}\right)$ $\leq c_{5}(k, n)(\mathrm{a}+1)^{n-1} P^{n \alpha-a \alpha}$. Here $a=1 / k$.

Theorem 3: Make $f\left(x_{1}, \cdots, x_{n}\right)$ be a polynomial of degree $k$ with integer coefficients, and assume that the greatest common divisor of all coefficients is 1 . Then 
$\sum_{x_{1}=1}^{P} \cdots \sum_{x_{n}=1}^{P} d^{l}\left(\left|f\left(x_{1}, \cdots, x_{n}\right)\right|\right) \leq c_{1}(k, n, l) \mathrm{A}(\log x)^{c_{2}(k, n, l)}$

$f\left(x_{1}, \cdots, x_{n}\right) \neq 0$

Here $x$ represents the maximum value of $\left|f\left(x_{1}, \cdots, x_{n}\right)\right|$ in $1 \leq x_{1} \cdots x_{n} \leq P, A=\max \left(\mathrm{P}^{n}, x^{n / k}\right)$.

\subsection{The conclusion to be proved in this part}

Theorem 4: Make $f\left(x_{1}, \cdots, x_{n}\right)$ be a polynomial of degree $k$ with integer coefficients, and assume that the greatest common divisor of all coefficients is 1 . Then when $1 \leq x_{1}, \cdots, x_{n} \leq q$, the solution number of $f\left(x_{1}, \cdots, x_{n}\right) \equiv 0(\bmod q) \leq 2^{n \pi(2 \mathrm{k})} \bullet q^{n-\frac{1}{k}} d_{(\mathrm{q})}^{n-1}$

Theorem 5: Make $f\left(x_{1}, \cdots, x_{n}\right)$ be a polynomial of degree $k$ with integer coefficients, and assume that the greatest common divisor of all coefficients is 1 . Then

$$
\begin{aligned}
& \sum_{x_{1}=1}^{P} \cdots \sum_{x_{n}=1}^{P} d^{l}\left(\left|f\left(x_{1}, \cdots, x_{n}\right)\right|\right) \leq c^{\frac{n}{k}} \cdot \mathrm{c}_{1}(k, n, l) \mathrm{P}^{n}(\log \mathrm{CP})^{c_{2}(k, n, l)} \\
& f\left(x_{1} \cdots x_{n}\right) \neq 0
\end{aligned}
$$

Among them, " $c$ " is the maximum value of the absolute value of each coefficient in $f\left(x_{1}, \cdots, x_{n}\right) \neq 0$.

\subsection{Proof of several lemmas}

Lemma 1: Make $f\left(x_{1}, \cdots, x_{n}\right) \neq 0$ be a polynomial with integer coefficients, and assume that not all of its coefficients are multiples of $P$. Then ${ }^{[8]}$

The solution number of $f\left(x_{1}, \cdots, x_{n}\right) \equiv 0\left(\operatorname{modP} \mathrm{P}^{\alpha}\right) \leq \mathrm{nkP}^{n \alpha-1}$.

The proving process is: When $n=1$, it is easy to know the solution number of $\left.f(x) \equiv 0(\operatorname{modP})^{\alpha}\right) \leq \mathrm{kP}^{\alpha-1}$ is obviously true. Suppose that when $n=k_{0}$, the solution number of $f\left(x_{1}, \cdots, x_{k_{0}}\right) \equiv 0\left(\operatorname{modP}^{\alpha}\right) \leq k_{0} k \mathrm{P}^{k_{0} \alpha-1}$. Next it will be proved that the conclusion is also true when $n=k_{0}+1$.

First write $f\left(x_{1}, \cdots, x_{k_{0}+1}\right) \equiv 0\left(\operatorname{modP}{ }^{\alpha}\right)$ into the form of $\left.g_{0} x_{k_{0}+1}^{s}+\cdots+g_{0} \equiv 0(\operatorname{modP})^{\alpha}\right)$, where $g_{i}=g_{i}\left(x_{1}, \cdots, x_{k_{0}}\right)$. It is easy to know that at least one of the coefficients in " $g_{i_{0}}$ " is not divisible by $P$. Otherwise all coefficients of $f\left(x_{1}, \cdots, x_{k_{0}+1}\right)$ are divisible by $P^{[7]}$.

(1) When $g_{i_{0}}\left(x_{1}, \cdots, x_{k_{0}}\right) \equiv 0\left(\operatorname{modP} \mathrm{P}^{\alpha}\right)$, the solution number $\leq \mathrm{k}_{0} \mathrm{kP}^{k_{0} \alpha-1} \cdot \mathrm{P}^{\alpha}=\mathrm{k}_{0} \mathrm{kP}^{\left(k_{0}+1\right) \alpha-1}$ is easy to know from the assumption.

(2) When $g_{i}\left(x_{1}, \cdots, x_{k_{0}}\right) \neq 0\left(\bmod \mathrm{P}^{\alpha}\right)$, for a certain set of values $x_{1}, \cdots, x_{k_{0}}$, the solution number of $f\left(x_{1}, \cdots, x_{k_{0}+1}\right) \equiv 0\left(\operatorname{modP}^{\alpha}\right)$ related to “ $x_{k_{0}+1}$ ” $\leq P^{\alpha-1}$.

From this it is easy to know: the solution number of $f\left(x_{1}, \cdots, x_{k_{0}+1}\right) \equiv 0\left(\operatorname{modP}^{\alpha}\right)$ $\leq\left(\mathrm{P}^{\alpha}\right)^{k_{0}} \cdot\left(\mathrm{kP}^{\alpha-1}\right)=\mathrm{kP}^{\left(k_{0}+1\right) \alpha-1}$ when $g_{i_{0}}\left(x_{1}, \cdots, x_{k_{0}}\right) \neq 0(\operatorname{modP})^{\alpha}$.

Based on the two cases (1) and (2), it can be known: the solution number of $f\left(x_{1}, \cdots, x_{k_{0}+1}\right) \equiv 0(\operatorname{modP})$ $\leq \mathrm{kP}^{\left(k_{0}+1\right) \alpha-1}+k_{0} \mathrm{kP}^{\left(k_{0}+1\right)-1}=\left(\mathrm{k}_{0}+1\right) \mathrm{kP}^{\left(k_{0}+1\right) \alpha-1}$.

That is: the conclusion holds when $n=k_{0}+1$. The proving process is completed.

Lemma 2: Under the conditions of Lemma 1,

The solution number of $f\left(x_{1}, \cdots, x_{k_{0}+1}\right) \equiv 0\left(\operatorname{modP}{ }^{\alpha}\right)$ 
$\leq \begin{cases}(\alpha+1)^{n-1} P^{n \alpha-a \alpha} & (\text { when } 2 k \leq P) \\ 2^{\alpha}(\alpha+1)^{n-1} P^{n \alpha-a \alpha} & (\text { when } 2 k>P \mid)\end{cases}$

The proving process is: it is easy to know from Lemma 2 and Lemma 3 in the third part that the solution number of $\left.f(x) \equiv 0(\operatorname{modP})^{\alpha}\right)$

$\leq\left\{\begin{array}{ll}P^{\alpha(1-a)} & (\text { when } 2 k \leq P) \\ 2 P^{\alpha(1-a)} & (\text { when } 2 k>P \mid)\end{array} \quad\right.$ Formula 1 )

That is: the conclusion holds when $n=1$; suppose the conclusion holds when $n \leq k_{0}$. It is now proved that the conclusion is also true when $n=k_{0}+1^{[9]}$.

First of all, write $f\left(x_{1}, \cdots, x_{k_{0}+1}\right)$ into the form of $f\left(x_{1}, \cdots, x_{k_{0}+1}\right)=g_{s} x_{k_{0}+1}^{s}+\cdots+g_{0}$. It is easy to prove that there is " $g_{i_{0}}$ ", and its coefficients are not all divided by " $P$ ".

(1) It is easy to know from the assumption: if $\lambda$ is a positive integer, the solution number of $P^{\lambda} \| g_{i_{0}}(1 \leq \lambda \leq \varepsilon-1)$ or $P^{\lambda} \mid g_{i_{0}}(\lambda=\alpha)$

$\leq c_{0}(\alpha+1)^{k_{0}-1} P^{k_{0} \lambda-\lambda \alpha} \cdot P^{k_{0}(\alpha-\lambda)}=c_{0} P^{k_{0} \alpha-\lambda \alpha} \cdot(\alpha+1)^{k_{0}-1}$.

Among them,

$c_{0}= \begin{cases}1 & (\text { when } 2 k \leq P) \\ 2^{k_{0}} & (\text { when } 2 k>P \mid)\end{cases}$

Under the condition of $P^{\lambda} \mid g_{i_{0}}(1 \leq \lambda \leq \alpha-1)$ or $P^{\lambda} \mid g_{i_{0}}(\lambda=\alpha)$, for a certain set of values $x_{1}, x_{2}, \cdots, x_{k_{0}}$ , the solution number of “ $f\left(x_{1}, \cdots, x_{k_{0}+1}\right) \equiv 0\left(\operatorname{modP} \mathrm{P}^{\alpha}\right)$ " related to " $x_{k_{0}+1}$ " is easy $\leq P^{\lambda} \cdot P^{(\alpha-\lambda)(1-a)} \bullet c_{1}$ $=c_{1} P^{a \lambda+\alpha-a \alpha}$

Among them,

$$
c_{1}= \begin{cases}1 & (\text { when } 2 k \leq P) \\ 2 & (\text { when } 2 k>P \mid)\end{cases}
$$

(2) When $g_{i_{0}} \neq 0(\operatorname{modP})$, it is easy to prove the solution number of $f\left(x_{1}, \cdots, x_{k_{0}+1}\right) \equiv 0\left(\operatorname{modP}^{\alpha}\right)$ $=c_{1} P^{\left(k_{0}+1\right) \alpha-a \alpha}$.

Based on the two cases (1) and (2), the solution number of $f\left(x_{1}, \cdots, x_{k_{0}+1}\right) \equiv 0\left(\operatorname{modP} \mathrm{P}^{\alpha}\right)$

$\leq \sum_{\lambda=1}^{a}\left(c_{0}(\alpha+1)^{k_{0}-1} P^{k_{0} \lambda-\lambda \alpha}\right) \cdot\left(P^{\alpha \lambda+\alpha-a \alpha} \cdot c_{1}\right)=\alpha c_{0} c_{1}(\alpha+1)^{k_{0}-1} P^{\left(k_{0}+1\right) \alpha-a \alpha}+c_{1} P^{\left(k_{0}+1\right) \alpha-a \alpha}$

$\leq \begin{cases}(\alpha+1)^{k_{0}} P^{\left(k_{0}+1\right) \alpha-a \alpha} & (\text { when } 2 k \leq P) \\ 2^{k_{0}+1}(\alpha+1)^{k_{0}} P^{\left(k_{0}+1\right) \alpha-a \alpha} & (\text { when } 2 k>P \mid)\end{cases}$

That is: the conclusion is also true when $n=k_{0}+1$. The proving process is completed.

Lemma 3: If let " $c$ " denote the maximum value of the absolute value of the coefficients in each term in $f\left(x_{1}, \cdots, x_{n}\right)$, then $^{[9]}$

$$
\left|\underset{1 \leq x_{i} \leq P, 1 \leq i \leq n}{\left(x_{1}, \cdots, x_{n}\right)}\right| \leq c(\mathrm{n}+1)^{k} P^{k}
$$

The proving process is: 


$$
\begin{aligned}
& \left.\mid \underset{1 \leq x_{i} \leq P, 1 \leq i \leq n}{f\left(x_{1}, \cdots, x_{n}\right)}\right) \mid \leq c \sum_{l_{1}+l_{1}+\cdots l_{n} \leq k} x_{1}^{h_{1}} x_{2}^{l_{2}} \cdots x_{n}^{l_{n}} \text { (li is a non-negative integer) } \\
\leq & c\left(x_{1}, x_{2}+\cdots+x_{n}+1\right)^{k} \leq c(\mathrm{nP}+1)^{k} \leq c(\mathrm{n}+1)^{k} P^{k}
\end{aligned}
$$

The proving process is completed.

\subsection{Proof of conclusions}

\subsubsection{Proof of Theorem 4}

Let, and then the solution number of $f\left(x_{1}, \cdots, x_{k_{0}+1}\right) \equiv 0$ (modq) can be obtained from Lemma 2:

$$
\begin{aligned}
& N \leq \prod_{i=1}^{\mathrm{s}}\left(\mathrm{c}_{2}\left(l_{i}+1\right)^{n-1} \mathrm{P}_{i}^{n l_{i}-a l_{i}}\right) \\
& \leq 2^{n \pi(2 \mathrm{k})} \cdot d^{n-1}(q)^{n-\frac{1}{k}}
\end{aligned}
$$

The proving process is completed.

Note: Let $f\left(x_{1}, \cdots, x_{n}\right)=\left(x_{1}+x_{2}+\cdots+x_{n}\right)^{k}$ and " $q^{\frac{1}{k}}$ " be an integer and not less than 2. It is easy to prove that the solution number of $f\left(x_{1}, \cdots, x_{n}\right) \equiv 0$ (modq) is not less than " $q^{n-\frac{1}{k}}$ ". It can be obtained from this: if the upper limit of its solution number is $M$, then $q^{n-\frac{1}{k}} \leq M \leq 2^{n \pi(2 \mathrm{k})} \cdot d^{n-1}(q) q^{n-\frac{1}{k}}$. The specific proof is omitted[10].

\subsubsection{Proof of Theorem 5}

The proving process is: according to Theorem 3 in Additive Theory of Prime Numbers, it is known that

$$
\begin{aligned}
& \sum_{x_{1}=1}^{P} \cdots \sum_{x_{n}=1}^{P} d^{l}\left(\left|f\left(x_{1}, \cdots, x_{n}\right)\right|\right) \leq c_{1}(\mathrm{k}, \mathrm{n}, l) \mathrm{A}(\log x)^{c_{2}(\mathrm{k}, \mathrm{n}, l)} \\
& f\left(x_{1}, \cdots, x_{n}\right) \neq 0 .
\end{aligned}
$$

It is known by Lemma 3: $x \leq c(\mathrm{n}+1)^{k} P^{k}$

$$
\begin{aligned}
& A \leq x^{n / k} \leq c^{\frac{n}{k}} \cdot(\mathrm{n}+1)^{n} P^{n} \\
& \Rightarrow \sum_{x_{1}=1}^{P} \cdots \sum_{x_{n}=1}^{P} d^{l}\left(\left|f\left(x_{1}, \cdots, x_{n}\right)\right|\right) \leq c^{\frac{n}{k}} \bullet c_{1}^{\prime}(\mathrm{k}, \mathrm{n}, l) \mathrm{P}^{n}(\log C \mathrm{P})^{c_{2}^{\prime}(\mathrm{k}, \mathrm{n}, l)}
\end{aligned}
$$

The proving process is completed.

Note: The original "Theorem 3" has two variables $A$ and $X$, and the maximum value of the complex multivariate function " $\left|f\left(x_{1}, \cdots, x_{n}\right)\right|$ " is very difficult to calculate. In this conclusion, " $c$ " is introduced to replace the two variables $A$ and $X$. In addition, the two conclusions of "Lemma 2.2" and "Lemma 2.3" cited in the original "Theorem 3" have been re-derived in this article, which may affect the specific expression forms of $c_{1}(\mathrm{k}, \mathrm{n}, l)$ and $c_{2}(\mathrm{k}, \mathrm{n}, l)$ in the original "Theorem 3". No more in-depth discussion will be done here.

\section{References}

1. Hua L. Additive theory of prime numbers. Beijing: Science Press; 1957.

2. Lv R. Research on factorization methods of the polynomial with integer coefficients. Chengdu: University of Electronic Science and Technology of China; 2008. doi: 10.7666/d.D310780.

3. Lu M. Some problems in additive number theory (I). Journal of Mathematical Research and Exposition 1984; (3): 115-124.

4. Wang M. On the mean value of natural number sequences and divisor functions (in Chinese). Natural Science Journal of Hainan University 2019; 37(4): 283-286.

5. Yang $X$. The average estimate of the divisor function of integer matrices on square-free numbers. Jinan: Shandong Normal University 2019; 35(1): 80-85. doi: CNKI:SUN:CCSX.0.2019-01-007. 
6. Xu J. The mean value of the arithmetical function $\mathrm{P}(\mathrm{n})$ in the set of total cubic numbers (in Chinese). Jinan: Shandong Normal University; 2016.

7. Fabrykowski J, Subbarao MV. The maximal order and the average order of multiplicative function $\sigma$ (e) (n). // Koninck JM, Levesque C. Number theory. Berlin: De Gruyter; 1989.

8. Li S, Dong L. The mean value of P(n) over square-full numbers. Scientia Magna 2014.

9. Ivić A, Shiu P. The distribution of powerful integers. Illinois Journal of Mathematics 1982; 26(4): 576-590. doi: $10.1215 / \mathrm{ijm} / 1256046597$. 